\title{
Filigrane
}

Écoutes psychanalytiques

\section{Expériences cliniques de la bisexualité}

\section{Jacques André}

Volume 28, numéro 1, 2019

Identités $^{2}$. Qui suis-je?

URI : https://id.erudit.org/iderudit/1064596ar

DOI : https://doi.org/10.7202/1064596ar

Aller au sommaire du numéro

Éditeur(s)

Santé mentale et société

\section{ISSN}

1192-1412 (imprimé)

1911-4656 (numérique)

Découvrir la revue

\section{Citer cet article}

André, J. (2019). Expériences cliniques de la bisexualité. Filigrane, 28(1), 43-56. https://doi.org/10.7202/1064596ar

\section{Résumé de l'article}

Les représentations sociales de la sexualité et les assignations et possibilités de genre ont été profondément modifiées depuis Freud, et plus intensément encore dans les dernières décennies. Qu'en est-il pourtant de l'expérience clinique du psychanalyste aujourd'hui et quelles conséquences théoriques peut-il en tirer ? Après avoir survolé les conceptions psychanalytiques classiques de la bisexualité psychique et avoir souligné le fait qu’une certaine identification a toujours lieu avant l'advenue et le « choix » du genre, quelques tableaux cliniques permettront de mettre en lumière diverses nuances dans l'expérience du genre, ainsi que la persistance d'angoisses et d'un certain destin du fantasme. L'élément le plus original de l'humaine sexualité demeure peut-être le fantasme, et le sexe le plus sexuel celui que ce fantasme imagine.
Ce document est protégé par la loi sur le droit d'auteur. L'utilisation des services d'Érudit (y compris la reproduction) est assujettie à sa politique d'utilisation que vous pouvez consulter en ligne.

https://apropos.erudit.org/fr/usagers/politique-dutilisation/ 


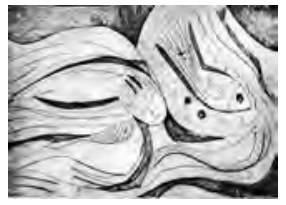

\title{
Expériences cliniques de la bisexualité
}

\author{
Jacques André
}

Résumé: Les représentations sociales de la sexualité et les assignations et possibilités de genre ont été profondément modifiées depuis Freud, et plus intensément encore dans les dernières décennies. Qu'en est-il pourtant de l'expérience clinique du psychanalyste aujourd'hui et quelles conséquences théoriques peut-il en tirer? Après avoir survolé les conceptions psychanalytiques classiques de la bisexualité psychique et avoir souligné le fait qu'une certaine identification a toujours lieu avant l'advenue et le "choix» du genre, quelques tableaux cliniques permettront de mettre en lumière diverses nuances dans l'expérience du genre, ainsi que la persistance d'angoisses et d'un certain destin du fantasme. L'élément le plus original de l'humaine sexualité demeure peut-être le fantasme, et le sexe le plus sexuel celui que ce fantasme imagine.

Mots clés: bisexualité; femme phallique; homosexualité; hétérosexualité.

\begin{abstract}
Social representations of sexuality and gender have profoundly changed since Freud, and even more intensely in recent decades. But what about the clinical experience of the psychoanalyst today, and what theoretical consequences can we draw from it? After having gone over the classic psychoanalytic conceptions of psychic bisexuality and underlined that a certain identification always takes place before the assumption and decision of the gendered self, some clinical cases will allow us to highlight various nuances in the experience of the gender, as well as the persistence of anxieties and a certain destiny of fantasy within gender identifications. The most original element of human sexuality may still have its roots in fantasy, and the most sexual sex might always be that which this fantasy imagines.
\end{abstract}

Key words: bisexuality; phallic woman; homosexuality; heterosexuality.

e m'habitue à concevoir chaque acte sexuel comme un processus entre quatre individus». Cette phrase de Freud qui entérine l'idée que la bisexualité psychique est la chose du monde inconscient la mieux partagée, est contemporaine de la naissance de la psychanalyse. 130 ans plus tard, après plus d'un siècle de «libération sexuelle» et une évolution des mœurs qui a profondément modifié les représentations sociales des homosexualités et des bisexualités, qu'en est-il de l'expérience du psychanalyste aujourd'hui et des conséquences théoriques que l'on peut en tirer? 
Le mot «homosexualité» en psychanalyse présente plus d'inconvénients que d'avantages, et d'abord celui d'unifier à partir d'une conduite sexuelle adulte des fonctionnements psychiques aussi pluriels que divers. Impossible de réduire les homosexualités à la seule condition du même sexe. "Homos», c'est-à-dire le même, le mot tombe parfois juste, quand le narcissisme et son jeu de doubles organisent les vies, mais ce n'est qu'un cas de figure.

Avec «hétérosexualité», c'est plus simple, tant l'usage du mot (créé tardivement, en écho à celui «d'homosexualité») ne s'accompagne en psychanalyse d'aucun souci conceptuel. Au fond ce mot est un pléonasme, s'il est vrai qu'il n'est rien de plus heteros, marqué par l'altérité, que l'humaine sexualité.

Il n'est pas sûr que la désignation «bisexualité» soit beaucoup plus satisfaisante. Là aussi, le premier inconvénient est d'unifier des configurations psychiques hétérogènes et de dire en un mot ce qu'il faudrait nuancer en mille. Dans ce texte, mon choix sera donc kaléidoscopique, convoquant mille tableaux cliniques, ou à tout le moins une diversité ayant notamment pour but de décaler les vies psychiques des comportements sexuels. Aujourd'hui - davantage même que dans un passé proche -, beaucoup plus d'hommes et de femmes font l'expérience de relations sexuelles avec des partenaires des deux sexes. Un certain nombre d'entre eux fréquentent le divan du psychanalyste. Dans quelle mesure les acquis théoriques s'en trouvent-ils modifiés?

D'abord un bref rappel des composantes les plus souvent sollicitées quand il s'agit de bisexualité psychique.

Les identifications les plus primaires pour l'enfant qui vient de naître sont celles dont il est l'objet, et notamment les identifications émanant de l'inconscient parental. On est identifié avant de disposer des moyens psychiques de s'identifier. On sait la profondeur de la trace laissée par ces mouvements primitifs, comme celle d'être assignée à la place de l'enfant de remplacement après la mort récente d'un aîné. Il en va de même pour le sexe, celui qui est attendu et désiré, éventuellement distinct de celui que la nature propose. Il peut en résulter une sorte de bisexualité «objective», quand à la fille tant espérée répond la naissance d'un garçon, ou l'inverse.

Envisagé du point de vue du sujet cette fois, le jeu combiné des désirs et des identifications est évidemment décisif. Le fantasme de scène primitive condense ces deux dimensions, l'identification à l'autre sexe n'étant pas moins prégnante que l'identification au même. D'une certaine façon, la bisexualité psychique peut s'entendre comme le destin inconscient de la scène primitive. 
Inévitable référence théorique quand il s'agit de bisexualité: le narcissisme. L'intégrité, la complétude dont celui-ci rêve trouve dans la bisexualité un étayage précieux, les deux sexes étant moins opposés que réunis comme les deux faces d'une même médaille.

Sans se confondre avec le point de vue narcissique, la référence à la castration emprunte un chemin proche, avec des nuances cependant. Ce n'est pas la même opération psychique de dénier la différence des sexes et de ne pas renoncer aux deux sexes dont on dispose, grâce à l'identification. J'essaierai de préciser cela à partir des figures cliniques.

Qu'il s'agisse de narcissisme ou de castration, la bisexualité épouse un mouvement centripète, contribuant à la construction du moi, voire à sa protection ou à sa défense. Une autre dimension théorique ouvre davantage sur un mouvement centrifuge, tant la bisexualité participe de la plasticité psychique. Ce n'est pas par hasard si elle est souvent associée aux conditions psychiques de la créativité. C'est l'idée d'une mobilité qui permet de se déplacer d'un sexe psychique à l'autre. Y compris dans la vie sexuelle; c'est ainsi que j'entends la phrase célèbre de Freud: «Je m'habitue à concevoir chaque acte sexuel comme un processus entre quatre individus». Est-il besoin de préciser à quel point cette plasticité nourrie de bisexualité est indispensable au fonctionnement psychique de l'analyste? Comment autrement pouvoir voyager dans le transfert et visiter, quand on est un homme, sa part d'homosexualité féminine et, quand on est une femme, son homosexualité masculine? Freud, lui-même, attribue après coup ses échecs avec trois de ses patients (Dora, la jeune homosexuelle et Ferenczi) à sa réticence à se laisser déloger d'une position paternelle et hétérosexuelle.

Remarque incidente: le fantasme est l'élément de la bisexualité psychique. Lorsque la réalité, un état inter-sexué à la naissance ou une demande chirurgicale de changement de sexe s'en mêlent, ce n'est jamais au profit de la plasticité psychique. Mais toujours inversement contre elle.

Quelques mots encore avant d'en venir aux tableaux cliniques. L'un des mérites du monde psychanalytique d'aujourd'hui et des patients «inédits» qu'il propose, est de faire vaciller quelques constructions et d'en révéler la nature de simple échafaudage. Un exemple. J'ai depuis assez longtemps été intrigué par la violence délétère mise en acte par certaines homosexualités masculines. C'est elle qui conduit Ambroise vers l'analyse. En marge de sa vie paisible et très asexuée avec son compagnon, il fréquente les backrooms. Il décrit la dernière scène à laquelle il s'est livré: ligoté nu sur une chaise, avant que la chaise ne soit renversée et que pleuvent les coups de pieds sur 
tout le corps... cette fois il a eu très peur, comment tout cela va-t-il finir? Comme Pasolini ou Versace?

Y aurait-il une sorte de lien électif entre homosexualité masculine et destructivité, comme une figure privilégiée de ce que Laplanche nomme «pulsion sexuelle de mort»? Sur cette piste, Freud a semé quelques indices, notamment à partir de sa lecture de la nouvelle de Zweig, La confusion des sentiments. Dans ce récit, écrit Freud, le conflit consiste "dans le fait que l'adolescent voudrait répondre à l'amour de l'homme, mais ne le peut pas à cause d'un mystérieux interdit intérieur.» En 1937 ( «Analyse avec fin, analyse sans fin »), il fait ce constat qui n'a pas pris une ride si l'on se fie à ce qui s'entend sur le divan d'aujourd'hui: il est des «êtres humains qui peuvent prendre comme objets sexuels des personnes de même sexe aussi bien que de l'autre, sans que l'une des orientations porte préjudice à l'autre.» (Freud, 2010, p. 45-46) Mais alors même que cette bisexualité concerne tout un chacun, le vécu de celle-ci est loin d'être homogène: pour certains

les deux orientations sont accordées sans heurt, elles se trouvent dans l'autre cas, qui est le plus fréquent, dans un état de conflit excluant toute conciliation. L'hétérosexualité d'un homme ne tolère aucune homosexualité, et vice versa. (Freud, 1937, p. 46)

Pourquoi la libido est-elle incapable de se partager chez ces derniers alors qu'elle y parvient sans peine chez les premiers? À quelle source puise une telle inclination au conflit? La réponse de Freud est surprenante. Les horreurs névrotiques ne manquent pas (celles que révèlent le dégoût, l'aversion, la répulsion...), et parce que l'inconscient ignore le temps, elles s'entendent aujourd'hui comme hier: horreur de l'homme hétérosexuel devant la féminisation par sodomie, horreur de la femme hétérosexuelle devant l'intimité féminine quand elle touche au sexe, horreur de l'homme homosexuel à la pensée gluante et abyssale du sexe/gouffre féminin, horreur de la femme homosexuelle à la pensée de la pénétration (quand bien même une autre homosexualité féminine n'imagine pas se passer du "phallus lesbien», le godemiché). Et pourtant ce ne sont pas ces angoisses névrotiques que Freud invoque dans ce texte clinique testamentaire, mais une complicité secrète entre le conflit psychique et les pulsions de destruction. L'antagonisme serait moins entre un désir sexuel et son refoulement, qu'entre l'intégrité du moi et la menace que fait peser un sexuel-de-mort, plus délétère qu'érotique. Ce climat trash avant l'heure n'est pas absent de la nouvelle de Zweig, quand il 
évoque l'homosexualité à la Pasolini du professeur, plus proche des bas-fonds et du meurtre que de l'hédonisme. Il n'est pas rare que l'homme homosexuel radicalise la distinction entre l'amour et la baise. Il en reste une trace dans ce qui différencie Tinder de Grindr. Quand bien même la séquence sexuelle est la visée implicite et partagée par les deux protagonistes du «match» Tinder, quelque chose des «formes» demeure: «tu fais quoi dans la vie, qu'est-ce que tu aimes, et si on prenait un verre...» Aucune de ces précautions oratoires sur Grindr (réseau spécifiquement gay) : les mots sont immédiatement argotiques, et seuls le sexe et l'orifice font l'objet de l'échange. «Tu baises comment et par où?» Partiellisation maximum de l'objet, disparition de l'objet total. Pulsion sexuelle de mort ou mise en scène sexuelle de la destructivité?

Ambroise me ferait pencher du côté de la première hypothèse, Armand du côté de la seconde. C'est un patient chez lequel l'homosexualité se conjugue avec des traits psychotiques, schizoïdes et paranoïdes. Parmi les captures d'écran qu'il collectionne à l'infini, il y a cette vidéo, probablement un snuff movie, sur laquelle il se masturbe jusqu'à la décharge: un poing frappe un visage. Le poing frappe régulièrement, toujours au même rythme, un visage qui, au fur et à mesure, perd ses traits, jusqu'à ne plus être qu'une bouillie. D'un côté, le poing n'est celui d'aucune personne identifiable. De l'autre, le visage est inversement celui d'une personne qui est progressivement détruite comme telle.

J'en étais là de ces réflexions clinico-théoriques intriquant les sexualités partielles, le narcissisme de mort, la destructivité et l'homosexualité masculine (ou la haine de l'homosexualité masculine, tant celle-ci pousse aussi de façon privilégiée au meurtre). Jusqu'au jour où j'ai reçu Adélaïde... Elle a vécu sans retenue l'expérience du Pulp, ce haut lieu de la nuit parisienne lesbienne qui a fermé en 2007. Musique, drogues, baises et surtout bagarres... Elle y a pris plus que sa part, a beaucoup baisé, s'est beaucoup battue. «Si on voulait faire pipi, dit-elle, il valait mieux traverser la rue et aller dans la boîte gay d'en face, parce que les toilettes du Pulp étaient un baisodrome sans relâche». Quand elle redescend un peu, qu'elle cherche le calme, elle noue une relation avec un garçon, «c'est tellement plus doux et plus tendre...» La destructivité, elle aussi, est paritaire.

\section{Deux séquences cliniques évoquant la bisexualité}

J'en viens aux séquences cliniques, en choisissant délibérément d'évoquer la bisexualité à partir de contextes différents et de vies qui peuvent être hétéro, homo ou bisexuelles. 


\section{Axiane}

Axiane est une jeune femme aussi belle que séduisante. Elle dispose d'une capacité à figurer et agir ce que je serais tenté d'appeler la bisexualité du féminin, avec une radicalité qui n'est pas ordinaire.

Entre minceur et maigreur, son corps parfois hésite, à l'image de la relation qu'elle entretient avec le syndrome anorexique. Elle partage bien des aspects du fonctionnement psychique de celui-ci, sans en subir les aspects délétères. Son poids, la circulation digestive, tout cela est soumis à un strict contrôle, mais sur un mode tempéré qui respecte la santé.

Sa belle verticalité est un hommage appuyé au phallus et à son érection permanente. À moins de 1,90 m, les hommes ne l'intéressent pas, ce dont étrangement l'amour de transfert ne souffre guère. La «force d'attraction » du transfert est une surprise toujours renouvelée. Axiane est assez convaincue que je suis «le plus grand»... des psychanalystes parisiens.

«N'avoir que la peau et les os», cette expression-repoussoir est à l'inverse une image qui la séduit. Elle aime sentir et voir ses os sous la peau. Même les muscles, dont elle soigne la fermeté, n'ont pas la dureté de l'os, seule véritablement capable de contenir ses angoisses concernant l'intérieur du corps. Parce que, pour Axiane et ses sœurs, le corps-fascinus est aussi inséparable d'un autre corps que le sont recto et verso d'une même feuille, un corps aussi intérieur qu'invisible, aussi informe qu'abyssal. L'affichage de l'un, phallus-fascinus, est à la fois le masque et le démenti de l'autre, fosse marine insondable.

L'éclat phallique commande plusieurs aspects de sa vie psychosexuelle. D'abord son style conquérant, en amour comme dans la vie professionnelle et transférentielle. Il arrive que ses premiers mots en s'allongeant sur le divan soient: «J'ai très envie de baiser.» De l'homme, partenaire sexuel, il est attendu non seulement qu'il soit grand mais aussi bien membré. En une occasion au moins, alors que la nudité de l'amant d'un soir s'était révélée inversement proportionnelle à la promesse de la stature, elle lui avait dit son impossibilité de poursuivre et l'avait envoyé se rhabiller. L'humour d'Axiane et son sens de la répartie sont les meilleures armes de son talent castrateur. Sans surprise, elle fait l'apologie du clitoris: «L'homme que vous êtes, ditelle, n'a pas idée de son extrême sensibilité, à peine touché et la porte du temple s'ouvre.» Empêtré dans sa représentation étroitement phalliciste de la féminité, le Freud de 1932, celui de la $33^{\text {e }}$ conférence, faute d'inscrire l'intérieur féminin dans le sexuel infantile et d'en envisager le possible refoulement, ne sait plus rendre compte de la frigidité autrement qu'en invoquant 
un activisme clitoridien qui ne veut pas céder la place. Axiane semble toute disposée à confirmer ce point de la théorie-fantasme de Freud, l'orgasme lors de la pénétration ne lui est effectivement pas garanti. Sauf qu'elle y parvient néanmoins, dans une jouissance qu'elle décrit comme démesurée, quand certaines conditions fantasmatiques sont réunies. Après la clarté du phallus, la nuit du dark continent. Jamais elle n'a connu un plaisir équivalant à celui qu'elle doit à un ex-compagnon et amant qui, à la suite d'une de leurs très violentes et fréquentes disputes, l'avait bousculée vers le balcon de leur appartement, lui avait arraché ses vêtements, et prise sans ménagement. Scène primitive qui mime et joue le viol d'aussi près que possible, $y$ compris dans la note exhibitionniste en offrant le spectacle à un (enfant) voyeur potentiel. Pour qu'advienne le vagin... mot qu'Axiane prononce avec réticence, qu'il devienne une zone érogène, «il faut, dit-elle, qu'il soit maltraité». Un enfant est battu n'est pas loin, et avec lui la représentation d'une féminité primitive conjuguant passivité et effraction.

Axiane conjugue la bisexualité au féminin pluriel, même si c'est au sein de la seule hétérosexualité qu'elle se déploie. Ses deux féminins, celui qui croit au Phallus comme le seul sexe digne de ce nom, et celui qui identifie la position féminine à l'être-violé, nourrissent des sexualités différentes. Phallique, elle conquiert et triomphe des hommes comme un homme peut le faire des femmes, en les rabaissant, en les féminisant. Ce qu'elle énonce aussi clairement que possible dans ce qu'elle attend du garçon qu'elle espère mettre au monde un jour: qu'il «baise toutes ces petites salopes». Et «violée», elle jouit comme jamais d'être pénétrée, tout en s'identifiant à l'agresseur, au «violeur».

Que l'assimilation de la vulve à une blessure ou une cicatrice constitue pour bien des hommes un écueil du féminin, toute une clinique du fiasco et de l'éjaculation précoce est là pour le confirmer. Que cette même équation nourrisse la problématique phallique de bien des femmes, Axiane et consœurs sont là pour le dire. De façon assez remarquable, c'est la fertilité elle-même qu'Axiane enrôle sous la bannière du phallus. Elle qui a deux jeunes enfants et aimerait bien en avoir un troisième, un garçon cette fois, se montre régulièrement inquiète à la moindre perturbation gynécologique de sa possible infertilité, traduite en possible impuissance. Parlant en son nom, j'ai pu lui dire un jour: «je suis fertile, donc je bande!» Ce qui la fit associer sur le plaisir récent que lui avait procuré une échographie montrant la forme arrondie nettement dessinée de ses follicules ovariens. "On a les couilles qu'on peut». 


\section{Nabal}

Nabal partage avec Axiane un rapport plutôt cru avec la sexualité, à la fois parce qu'il en va ainsi de l'air du temps mais aussi parce que l'un et l'autre entretiennent avec la chose inconsciente une certaine proximité, voire une certaine vérité. Chez Nabal aussi, le féminin se décline au pluriel. Le féminin-châtré est le premier à se faire entendre. Féminin-châtré, c'està-dire tout en négativité, un féminin par défaut, fait de ce qui manque: la force, la virilité, les centimètres... La castration chez lui souffre de réalité; il n'a pas «le paquet», la tenue en maillot de bain lui est insupportable, elle qui exhibe aux yeux de tous son sexe d'enfant. C'est toujours un embarras quand la réalité obère ainsi la réalité psychique. Sauf que, à ma propre surprise, ce thème d'abord lancinant va assez rapidement s'effacer au rythme simultané du progrès de l'analyse et de l'évolution de sa vie amoureuse et sexuelle. De même pour son symptôme principal, l'éjaculation précoce, dont il cessera assez rapidement de se plaindre.

Ce pénis qu'il n'a que trop peu, il l'admire chez d'autres et ne se prive pas pour ce faire de quelques moments sexuels, qu'il pratique la fellation sur un partenaire d'un soir ou qu'il se fasse sodomiser. Moments sexuels, jamais amoureux, l'idée d'une relation avec un autre homme (pour ça il y a les amis) lui est plus étrangère que désagréable. C'est bien le sexe-phallus qu'il n'a pas qu'il vénère par la fellation chez un autre homme mieux pourvu, et non le même (homos) sexe que le sien. On mesure encore à quel point le mot convenu d' «homosexualité» manque la réalité psychique concernée. C'est encore plus vrai sous l'angle de la sodomie, qui engage chez lui une autre figure de la féminité, celle qui se définit par être-pénétré et non plus par être-châtré.

Nabal vivra d'abord sa bisexualité sur un mode conflictuel et malheureux, beaucoup par condensation de sa féminité avec les représentations de la castration, condamnant cette sexualité avec un homme à souligner sa faiblesse, et cela malgré les satisfactions qu'il peut en tirer. La sortie du conflit prendra la forme d'un énoncé libératoire: «Je sais maintenant que je peux être homosexuel, donc je n'ai pas besoin de l'être.» L'idée donc d'une possibilité offerte, qu'il n'est pas nécessaire de combattre, qui n'entre pas dans une opposition binaire, toujours au bout du compte phallique: être homo ou hétéro, c'est l'un ou l'autre... L'accès aux femmes ne suppose pas le renoncement aux hommes. Être une fille ce n'est pas: ne pas être un garçon... Quel chemin a-t-il parcouru pour parvenir à cette liberté nouvelle? Je pourrais bien sûr faire l'inventaire des passages probables. Ce qui est plus 
vrai c'est qu'au fond je n'en sais rien, avec cependant une conviction, celle que la passivité actualisée par le transfert, son processus de féminisation, a joué un rôle important. "Je peux être homosexuel», doit sans doute s'entendre: je peux être homosexuel ici avec vous, je peux me laisser pénétrer par vous, parce que vous-même pouvez y consentir sans en être angoissé. La condition pour qu'un analysant accepte la passivité devant un homme-analyste est certainement que ce dernier puisse tolérer de pénétrer son patient. Parce qu'il est plus facile de l'entendre chez un autre que chez soi-même, le travail de supervision avec un analyste-homme donne fréquemment à entendre la façon dont celui-ci se défend contre-transférentiellement de la scène homosexuelle par des évitements ou des interprétations défensives.

La pénétration de l'adolescent passif, éromène, par l'adulte actif, éraste, est un passage obligé de la transmission de la virilité dans la Sparte antique et guerrière. La féminisation est un préliminaire à la virilisation, et non son contraire. Se pourrait-il qu'il en ait été ainsi pour Nabal? Le constat du début de cure, qu'il est beaucoup plus aisé de se laisser séduire par un homme dans un bar que de partir à la conquête d'une femme, va céder la place à une position virile plus assurée au gré d'aventures hétérosexuelles gratifiantes. Son propre féminin, non plus le féminin-châtré mais celui qui le fait s'identifier à la position féminine sur la scène primitive, ce féminin-là n'est pas pour Nabal un obstacle; celui de la femme à laquelle il prête une attente désirante insatiable, l'est bien davantage. À côté de Jocaste, Edipe est un petit garçon. Nabal est volontiers porté à ramener «l'insatiable» à sa source orale primitive, c'est un passionné du cunnilingus. Si sa bouche, dit-il, devait renoncer à l'un des deux plaisirs, fellation ou cunnilingus, il renoncerait au premier sans une seconde d'hésitation. L'enfant en naissant quitte le pays natal la tête la première, le cunnilingus emprunte le même chemin en sens inverse.

Le vécu d'aujourd'hui permet de réviser le caractère convenu de certaines formulations psychanalytiques. Pas de cure terminée, disait-on, sans qu'ait été analysée l'homosexualité inconsciente. Comme si l'homosexualité ne pouvait être que l'inconscient par excellence. C'est un mérite de l'époque de rappeler, s'il était nécessaire, toute l'âpreté passionnelle et conflictuelle de la relation homme/femme. Parce qu'elle n'est plus la seule union sociale possible, parce que l'union inclut la possible séparation, la relation hommefemme essuie plus que jamais les angoisses nées de la liberté psychique.

Nabal observe lui-même que les fantaisies «homo», voire la tentation du bar nocturne, ressurgissent quand «ça ne va pas très fort». Position passive vis-à-vis d'un autre homme et position dépressive naviguent de concert. 
La passivité a les accents du repli, du refuge, elle le protège; alors que la rencontre avec la femme et son altérité est toujours inquiétante. La note de la réparation narcissique est perceptible. Quand ça ne va vraiment plus du tout, c'est à l'auto-fellation au fond de la couette qu'il se livre, geste d'enclos sur lui-même qui confond la bouche et le vagin, le sperme et le lait, accomplissant le fantasme dépressif par excellence, celui du retour au ventre maternel.

Choisir pour Axiane et Nabal deux noms extraits du théâtre de Racine est sans doute une façon de chercher l'a-temporalité de l'inconscient derrière la vie sexuelle d'aujourd'hui. Chacun fait entendre à sa manière le caractère restrictif du mot «bisexualité». Bi, c'est seulement deux, c'est peu. Non seulement parce que chaque sexe est pluriel, mais aussi parce que c'est un relatif, $\mathrm{y}$ compris dans le geste onaniste, et que cette dimension relative contribue à dessiner le sexe en question: la vulve qu'explore la langue de Nabal n'est pas la même que celle qu'il aime tant observer quand sa partenaire est $a$ tergo. L'élément le plus original de l'humaine sexualité est le fantasme, et le sexe le plus sexuel celui que ce fantasme imagine. À la rigueur le mot «cul» sonne plus juste: mot confus, tout autant devant que derrière, dont on ne sait exactement où il commence et où il finit. Le «cul» est bisexuel, et même davantage.

\section{Trois figures cliniques pour conclure, trois visages de la bisexualité \\ Amalia}

Amalia est une jeune femme, lesbienne, d'origine uruguayenne. Quel est son genre? Elle évoque moins un garçon qu'un garçon travesti en fille. Son père (sa mère aussi, plus obscurément) désirait un garçon, il $a \mathrm{eu}$ un garçon - que le démenti par la perception échoue à produire son effet et l'empreinte du fantasme inconscient du parent dessine la réalité de l'enfant. Le choix amoureux d'Amalia est fidèle à cette première identification dont elle a été l'objet, elle aime l'autre sexe que son sexe psychique (et non pas le même sexe que le sien, le mot «homosexualité» la manque). Il y a pourtant un reste, elle se souvient de sa colère à la naissance de son petit frère, le jour de la circoncision. La preuve du pénis c'est qu’on peut le couper. «Moi aussi!», exige-telle. «Mais ce n'est rien qu'un petit bout de peau», lui avait dit le père. «Si c'est rien que ça, pourquoi pas moi?» Toute la vie d'Amalia est ponctuée de petits bouts, à l'image de l'angoisse qui l'avait assaillie quand elle avait 
perdu un point à son permis de conduire. Difficile d'être plus fortement convaincue que ne l'est Amalia par la théorie sexuelle infantile du primat du phallus. On l'a ou on ne l'a pas. C'est l'occasion de rappeler que l'on ne saurait confondre la critique idéologique et politique du binarisme, le politiquement correct d'aujourd'hui, avec une modification correspondante des formations de l'inconscient. Frédéric est un homme politique, occupant de hautes responsabilités, que l'on ne prendra jamais publiquement en défaut s'il s'agit de défendre la parité homme-femme. Il est tout heureux du bon mot qu'il s'entend dire sur le divan: "Il y a deux sexes, les hommes et les secrétaires.»

Conclure à une identité de genre masculine chez Amalia ferait l'impasse, non seulement sur sa bisexualité, même si celle-ci porte aussi la marque de la masculinité: quitte à être pénétrée, c'est à la enculada qu'elle s'en remet. Mais aussi l'impasse sur une complexité à laquelle participe le conflit psychique chez le père entre ce qu'il a vu et ce qu'il a voulu. Toute son enfance, il a appelé son enfant «Macha». Comme "macho», certes, sauf qu'un petit bout de lettre maintient la différence et le souvenir de la chose vue. «Sexe psychique», plus que "genre», me semble avoir quelque chance de nommer cet imbroglio dont la chose vue est toujours partie prenante.

\section{Florent}

La vie sexuelle de Florent ne fait pas partie des motifs qui le conduisent vers l'analyse. C'est un homme de spectacle qui remplit les salles parisiennes les plus connues, mais est assailli par l'angoisse de la salle vide, guettant régulièrement sur internet le taux de remplissage. Sa vie sexuelle est en parfait accord avec le fantasme du plus général des rabaissements, celui qui joue de l'opposition entre la Madone et la Putain, et distingue jusqu'au clivage le tendre et le sensuel. Fantasme masculin, néanmoins présent sur bien des scènes psychiques féminines, et surtout fantasme aussi «incorrect» qu'atemporel. Il ne constitue dans le fond qu'une variation sur le thème fantasmatique de la scène primitive. La "putain» en psychanalyse, c'est celle qui, à l'aube, promet tout l'amour du monde, et la nuit couche avec un autre homme, généralement le père.

Pour la mère de ses enfants, Florent a beaucoup d'estime et très peu de désir. Sa sexualité, il la vit avec ses maîtresses. Il aime les maltraiter, gestuellement et verbalement, même si les coups de pied qu'il leur donne restent imaginaires. L'ennui le gagne vite si la partenaire est «vaginale», il entend 
par là une femme soucieuse d'obtenir son propre plaisir et peu portée sur la sodomie.

De temps en temps, une fois par mois, il prend rendez-vous avec un prostitué homme auquel il impose toujours le même scénario: celui du rabaissement de la femme, lui en la circonstance, en se faisant sodomiser et insulter. Il se charge d'écrire les dialogues. Cette activité marginale reste secrète, et nécessite une petite ligne de coke pour pouvoir être réalisée. Bisexualité donc, entièrement organisée cette fois autour des deux faces d'un même fantasme, en variant simplement les rôles.

Quelques années après la fin de l'analyse, Florent reprendra rendez-vous, dans un moment de fragilité qui, à la fois, évoque son angoisse première et bouge les lignes de sa vie sexuelle. Il s'est séparé de son épouse et vit avec une femme qu'il aime autant qu'il baise. Cette levée du clivage le déstabilise, d'autant plus qu'il est pour elle sans secret et que tous les deux se permettent de temps en temps une partie à trois où il accomplit sur la même scène les deux moments actif et passif. La conséquence psychique la plus présente est la façon dont son angoisse se précise en angoisse de perte d'amour. Il anticipe la détresse qui serait la sienne si celle «qui lui donne tout» venait à le quitter.

\section{Lucie}

Si j'ai gardé Lucie pour la fin, c'est qu'elle ne vit ce qu'elle vit que parce que le monde d'aujourd'hui le lui permet, et qu'en même temps elle met au jour les ingrédients d'une sexualité qui n'a pas d'âge, si ce n'est celui de l'humaine sexualité.

C'est une jeune femme que la bisexualité divise presque à parité, sans que la démocratie y soit pour grand chose. Le trouble dans le genre auquel invite notre monde occidental l'autorise à vivre un moment sexuel ou une relation amoureuse, tant avec un homme qu'avec une femme. Elle est aussi $b i$ sur le terrain des générations, pouvant aimer un homme ou une femme dont l'âge évoque celui de ses parents, comme un ou une partenaire de sa classe d'âge. Un peu comme si toutes les possibilités de la palette œedipienne lui étaient demeurées accessibles. La problématique de la castration ne lui est pourtant pas étrangère, mais elle ne prend pas chez elle la forme d'un «l'avoir ou pas», mais plutôt d'un "pourquoi un seul sexe et pas les deux?» L'angoisse de perte d'amour jouera un rôle décisif dans le choix auquel elle finira par se restreindre: "Avec un homme, dit-elle, viendra inéluctablement le moment où il se tournera vers une femme plus jeune.» Au terme de nombreuses péripéties sexuelles et amoureuses, Lucie a donc établi une 
relation durable avec une femme de son âge. Très vite, elle fait partager à sa compagne son désir d'enfant. S'en suit une recherche méandreuse de la meilleure voie reproductive. Si elle avait pu donner corps aux tenants de son désir, c'est à un de ses amis, «beau et intelligent», qu'elle aurait demandé ce service. Cette voie, conforme au fantasme, est cependant apparue à Lucie et à sa compagne comme trop encombrée d'ennuis à venir, à commencer par l'impossible contrôle d'un sentiment de paternité chez l'homme en question. C'est donc au don de gamète et à la procréation médicalement assistée (PMA) à l'étranger que les deux femmes se sont résolues. Le pays choisi, le Danemark, l'a été parce qu'il permet l'éventuelle levée de l'anonymat du donneur, sinon un ami, au moins un homme connu. Une telle entreprise relève du parcours du combattant, on avait bien prévenu Lucie du faible taux de réussite, $30 \%$... Elle est enceinte à la première tentative, se pourrait-il que la force du transfert s'applique aussi à la PMA? Elle souhaitait un garçon, son vou sera exaucé, réalisant en quelque sorte sa bisexualité en donnant naissance à la part masculine d'elle-même, et tenant en même temps à distance son angoisse d'un «trop de femme».

Le choix d'évoquer Lucie est directement lié au débat avec les gender studies. Vu de loin, elle est près d'accomplir l'un des fantasmes organisateurs de la théorie/idéologie du genre: «Le rêve qui me semble le plus attachant, écrit Gail Rubin, est celui d'une société androgyne et sans genre (mais pas sans sexe) où l'anatomie sexuelle n'aurait rien à voir avec qui l'on est, ce que l'on fait, ni avec qui on fait l'amour.» (Rubin et Butler, 2001, p. 76) Vue de près, elle n'échappe à aucun moment au déterminisme inconscient de sa vie psycho-sexuelle et à l'intensité de son double investissement œedipien. Un déterminisme qui n'exclut pas la plasticité, laquelle se retrouve à l'œuvre dans la création artistique qui constitue aussi son métier.

C'est avec un mélange d'étonnement et d'humour que Lucie constate la façon dont la division des genres les rattrape, elle et sa compagne, alors que tout de leur vie sexuelle et sociale témoignait jusque-là d'une liberté maximale quant à l'assignation culturelle des rôles. Elle a été la femme enceinte et la mère allaitante, réjouie (et angoissée) d'un état comme de l'autre. Mais c'est sa compagne qui se révèle la-mère-de-bébé la plus investie; quand elle, Lucie, ne cache pas son impatience à retrouver pleinement sa vie sociale intense, car il faut bien ramener de l'argent à la maison...

Jacques André andre.jac@orange.fr 
56 Filigrane, 2019

\section{Références}

Freud, S. (2010). Analyse avec fin, analyse sans fin. Dans Euvres complètes XX. Paris: Presses universitaires de France.

Rubin, G. S. et Butler, J. (2001). Marché au sexe. Paris: EPEL. 